\title{
Teleradiology quality assurance - lessons learnt
}

\author{
Saskia Spijker
}

Received: 21 November 2013 / Accepted: 6 February 2014

(C) Springer-Verlag Berlin Heidelberg 2014

Quality assurance in radiography is an important process in identifying weaknesses in radiographic practice. Understanding where improvements can be made helps to keep the focus on those aspects of radiology where intervention can improve image quality. Quality assurance of radiographic images is also feasible for teleradiology platforms, and within Médecins Sans Frontières (MSF) all radiographic images sent for teleradiology are evaluated for quality (Fig. 1). Analysis of the image quality has proved useful in guiding the planning and prioritisation of radiology services within MSF. The lessons learnt may also useful for other users of radiography in resource-limited settings.

Comparing computed radiography images with those of film and chemistry, it is clear that computed radiography images are consistently of significantly higher quality [1]. This observation suggests that wherever possible, transitioning to computed radiography imaging can improve image quality compared to film and chemistry. This is partly because computed radiography technology can compensate for variables such as exposure and film processing. MSF sites that demonstrate better quality with computed radiography imaging have also had radiographer training as a part of the computed radiography installation. Good radiographic technique is a fundamental factor in improving image quality, and radiographer training, whether with computed radiography imaging or film and chemistry, results in better image quality. Wherever possible within MSF, radiographer training is prioritised and then regular visits are planned for continuation of training.

A point of discussion at MSF is whether to continue utilisation of referral radiology facilities where the image quality has proved to be consistently poor. If MSF is not in a position to upgrade equipment or provide an investment in radiographer training, it should be considered whether it might

\section{S. Spijker}

Médecins Sans Frontières International,

Amsterdam, the Netherlands

\section{S. Spijker $(\bowtie)$}

Plantage Middenlaan 14, Amsterdam 1018 DD, the Netherlands

e-mail: saskiaspijker@outlook.com

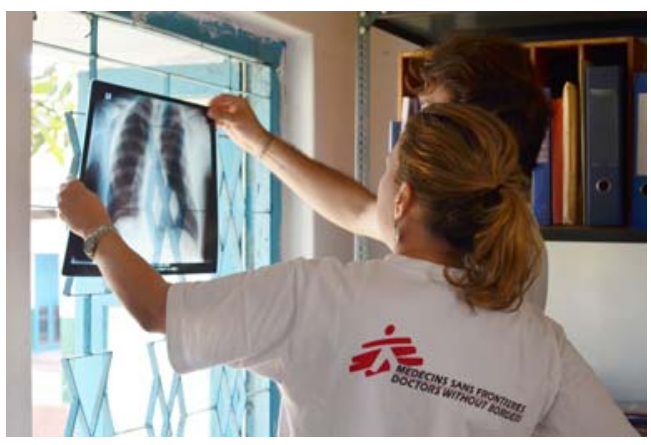

Fig. 1 A digital radiograph is viewed at a Médecins Sans Frontières project in Asia

be detrimental to patient outcomes to consult images that are very poor in diagnostic quality.

Further, MSF has learnt a few other lessons along the way. The teleradiology platform itself must be user-friendly and not require complex processes that require too much time or too many steps. If it's not straightforward, clinicians might find other avenues to ask for advice. Conversely, feedback to volunteer radiologists is very important in maintaining their interest and in respecting their time and investment in the outcome of the patient. Practically, this has been a difficult point for MSF to promote among field clinicians because their high workload, remote conditions and competing priorities leave less time for follow-up with consultants than clinicians would otherwise like. Last, we have found that radiologists' reports should attempt to help with a differential diagnosis as much as possible beyond just reporting radiologic findings in order to provide the most benefit to clinicians.

Conflicts of interest None

\section{References}

1. Spijker J, Andronikou S, Kosack C et al (2014) Quality assessment of $\mathrm{X}$-rays interpreted via teleradiology for Médecins Sans Frontières. J Telemed Telecare. doi:10.1177/1357633X14524153 